\title{
Une structure en pierres à caractère monumental à Incoronata
}

La campagne de 2020

\section{Mario Denti}

\section{CpenEdition} Journals

Édition électronique

URL : https://journals.openedition.org/baefe/2336

DOI : $10.4000 /$ baefe.2336

ISSN : 2732-687X

Éditeur

ResEFE

Référence électronique

Mario Denti, « Une structure en pierres à caractère monumental à Incoronata » [notice archéologique], Bulletin archéologique des Écoles françaises à l'étranger [En ligne], Italie, mis en ligne le 22 mai 2021, consulté le 24 mai 2021. URL : http://journals.openedition.org/baefe/2336 ; DOI : https://doi.org/ $10.4000 /$ baefe. 2336

Ce document a été généré automatiquement le 24 mai 2021.

\section{cc) $(1) \odot$}

Le Bulletin archéologique des Écoles françaises à l'étranger est mise à disposition selon les termes de la Licence Creative Commons Attribution - Pas d'Utilisation Commerciale - Pas de Modification 4.0 International. 


\section{Une structure en pierres à caractère monumental à Incoronata}

La campagne de 2020

\section{Mario Denti}

\section{NOTE DE L'AUTEUR}

Date précise de l'opération : 31 août-27 septembre 2020

Autorité nationale présente : Soprintendenza Archeologia Belle Arti e Paesaggio della Basilicata - MiC

Numéro de mission : Concession de fouilles MIBACT DGABAP Prot. n. 753 du 9/6/2020. Composition de l'équipe de terrain : Guilhem Chapelin, Centre Jean Bérard, USR 3133 - CNRS/EFR, architecte ; Chloé Damay, Laboratoire LAHM, UMR 6566 CReAAH, Université Rennes 2, archéologue ; Mario Denti, Laboratoire LAHM, UMR 6566 CReAAH, Université Rennes 2, IUF, archéologue ; Pierre Frétay, Université Rennes 2, étudiant ; Francesca Ippolito, University of Groningen, archéologue ; Josipa Mandić, Laboratoire LAHM, UMR 6566 CReAAH, Université Rennes 2, archéologue ; Lisa Marchand, LabEx Archimede, Université Paul Valéry, Montpellier 3, et Laboratoire LAHM, UMR 6566 CReAAH, Université Rennes 2 ; Clarisse Prêtre, CNRS, Laboratoire ANHIMA, UMR 8210, archéologue ; Giulia Saltini Semerari, Museum of Anthropological Archaeology, University of Michigan, Ann Arbor, archéologue ; Cesare Vita, Laboratoire LAHM, UMR 6566 CReAAH, Université Rennes 2, archéologue ; Chantal Willborn, Université Rennes 2, archéologue.

Partenariats institutionnels : CJB, Université Rennes 2, UMR 6566 CReAAH, IUF Établissement éditeur : CJB Établissements porteurs de l'opération : CJB, Université Rennes 2, UMR 6566 CReAAH, Laboratoire LAHM, IUF 
Données scientifiques produites :

https://centrejeanberard.cnrs.fr/spip.php?article342\&lang=fr

Chroniques de l'EFR :

https://journals.openedition.org/cefr/3534

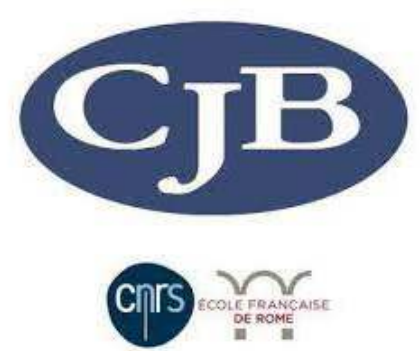

\section{Introduction}

1 Malgré les difficultés causées cette année par la crise sanitaire du Covid-19, la dix-huitième campagne de fouille à Incoronata a pu être organisée et menée à bon terme, dans un triple objectif : préserver la continuité scientifique de nos recherches; assurer l'activité et la permanence de la mission dans le territoire d'appartenance de la fouille et auprès de la communauté locale (Comune di Pisticci); garantir le maintien d'une perspective de recherche et d'implication forte pour les jeunes membres de notre équipe dans ce moment si difficile.

2 La mission a été organisée en nombre réduit, avec comme cible d'aborder, et d'essayer de résoudre, des questions stratigraphiques et contextuelles ponctuelles. Nous avons pris garde de garantir toutes les mesures sanitaires nécessaires sur le terrain, dans le laboratoire, aussi bien que dans la vie quotidienne de l'équipe. Comme chaque année, une conférence pour la diffusion des résultats de la fouille auprès de la communauté locale a été tenue dans la ville de Marconia.

3 Sur la colline d'Incoronata nous avons poursuivi l'exploration des contextes précédemment mis au jour dans la partie nord du secteur sud-occidental du plateau (Secteur 1), en ouvrant une surface de fouille de m $20 \times 10$ environ (fig. 1-2) dans l'objectif de poursuivre l'investigation stratigraphique des structures, des espaces et des bâtiments dont nous connaissions les niveaux d'utilisation les plus récents. 
Fig. 1. Planimétrie de la fouille, avec le marquage (en rouge) de la surface de l'intervention.

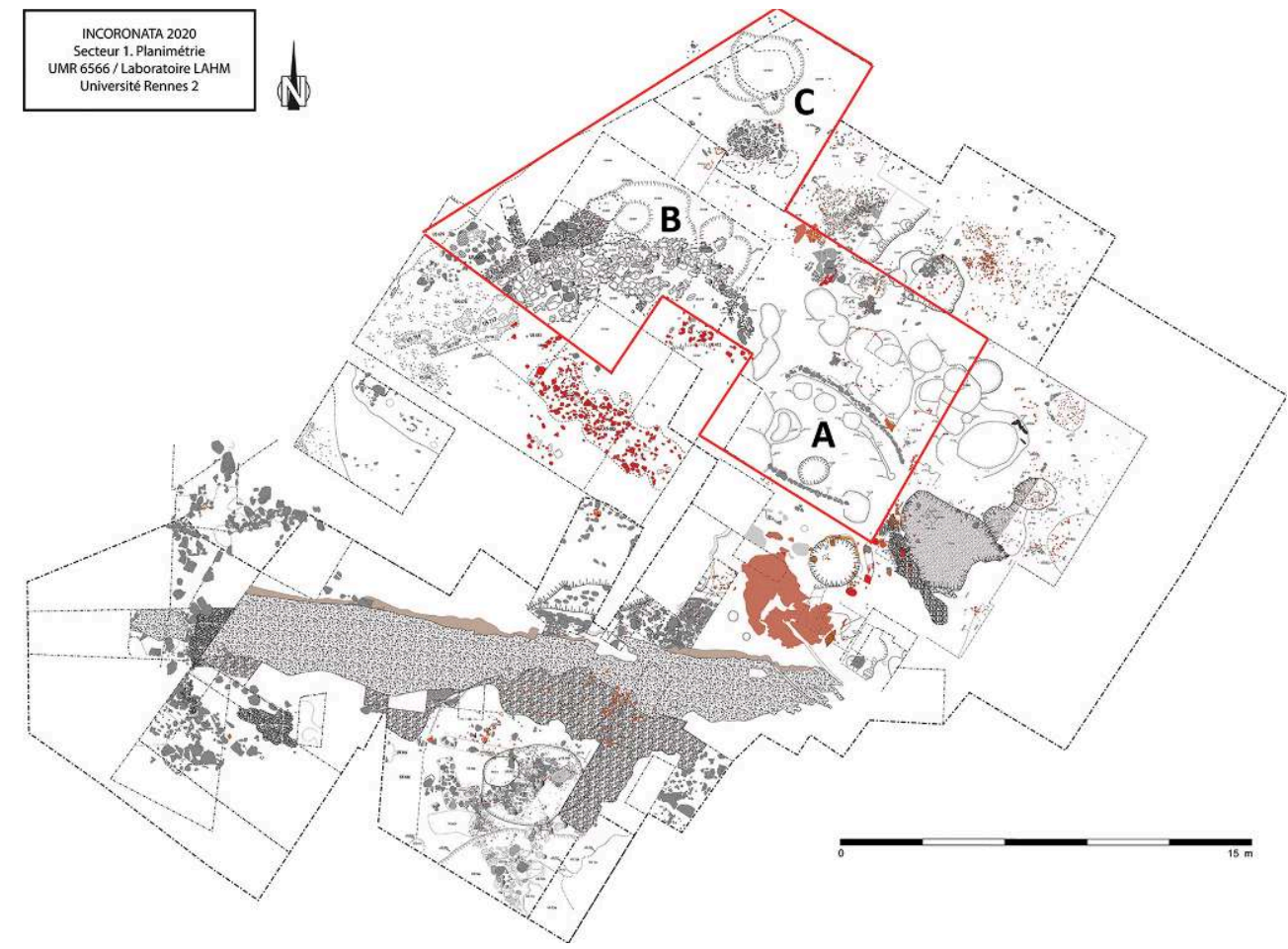

DAO T. Lisa Marchand.

Fig. 2. Vue aérienne du secteur fouillé.

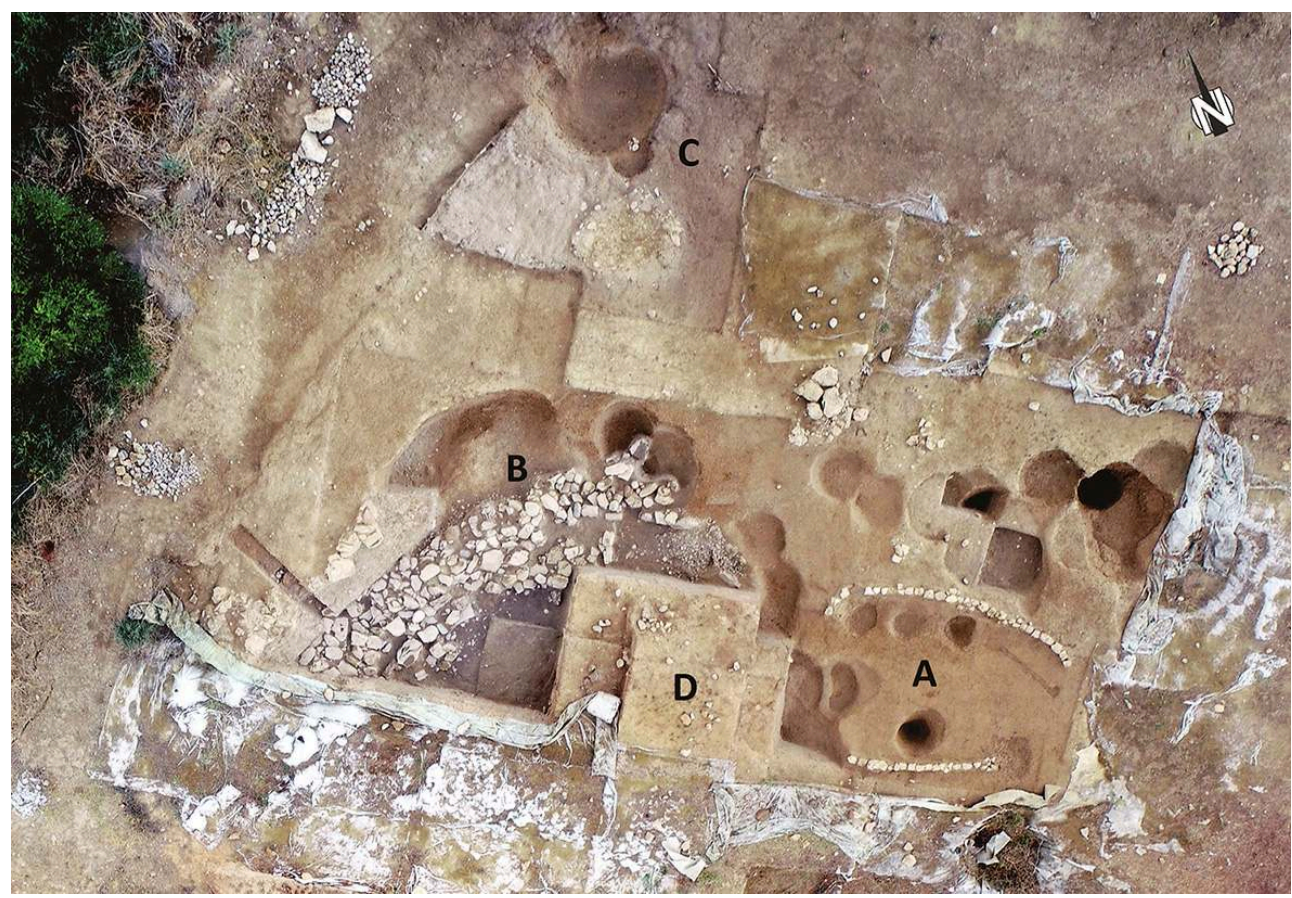

C. Vita. 


\section{L'édifice à abside}

4 L'une des questions primordiales concernait la compréhension des phases d'utilisation et de la chronologie de l'édifice à abside (fig. 1, A ; fig. 2, A). À l'intérieur, nous avons procédé à l'exploration des niveaux situés au-dessous du dernier plan de fréquentation, datable à la fin du VII e siècle, là où nous avions mis au jour, au milieu de l'abside, les restes de la dernière action rituelle attestée. Celle-ci, à caractère chtonien, comprenait une déposition de céramique œnôtre et grecque délimitée par un lit de micro galets (fig. 4, US 389) ${ }^{1}$.

5 Au-dessous de ce plan, sur le côté nord, 3 fosses parfaitement circulaires, de la même taille et peu profondes, étaient déjà apparues en 2019, alignées contre la paroi septentrionale de l'édifice (fig. 3, A, B, C; fig. 4, US 752, 751, 764) ${ }^{2}$. Ici, une petite canalisation, courant tout au long de la partie antérieure de cette paroi, a été mis au jour (fig. 3, D ; fig. 4, US 786, 787); elle terminait, à l'est, dans une concentration de gros galets fixés verticalement dans ce sol. Sur le côté sud, deux autres fosses ont été interceptées et fouillées: l'une peu profonde (fig. 3, E; fig. 4, US 784), l'autre très profonde (fig. 3, F ; fig. 4, US 794) contenant, parmi des petits tessons de céramique matt painted monochrome, un tesson à impasto noir lisse datable du VIII ${ }^{\mathrm{e}}$ siècle et, au milieu de la partie haute et de la partie basse de son remplissage, une strate de galets de taille petite et moyenne, dont une grande partie brisée intentionnellement.

Fig. 3. Photogrammétrie de l'édifice à abside.

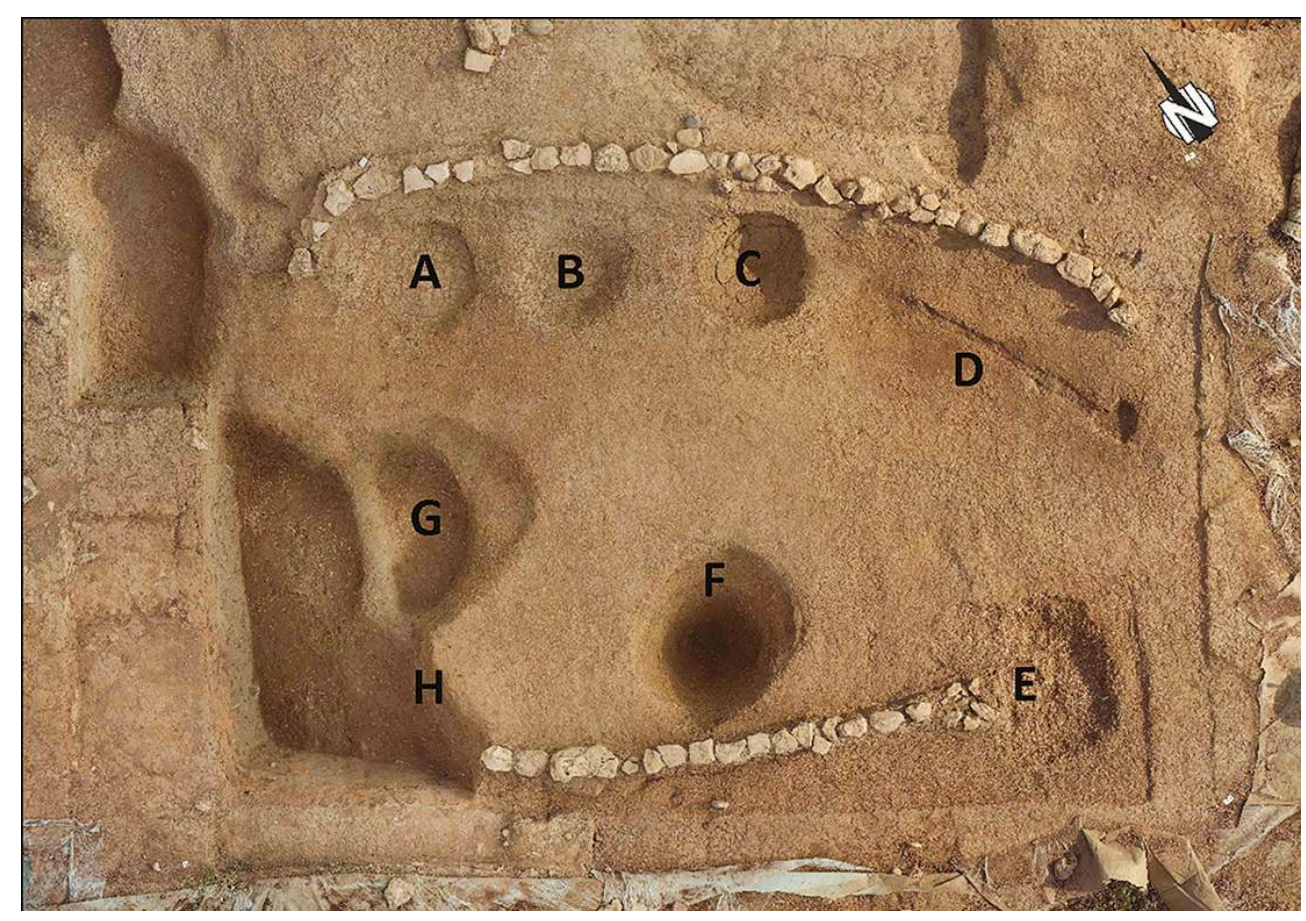

C. Vita. 
Fig. 4. Planimétrie cumulée 2013 et 2020 de l'édifice à abside.

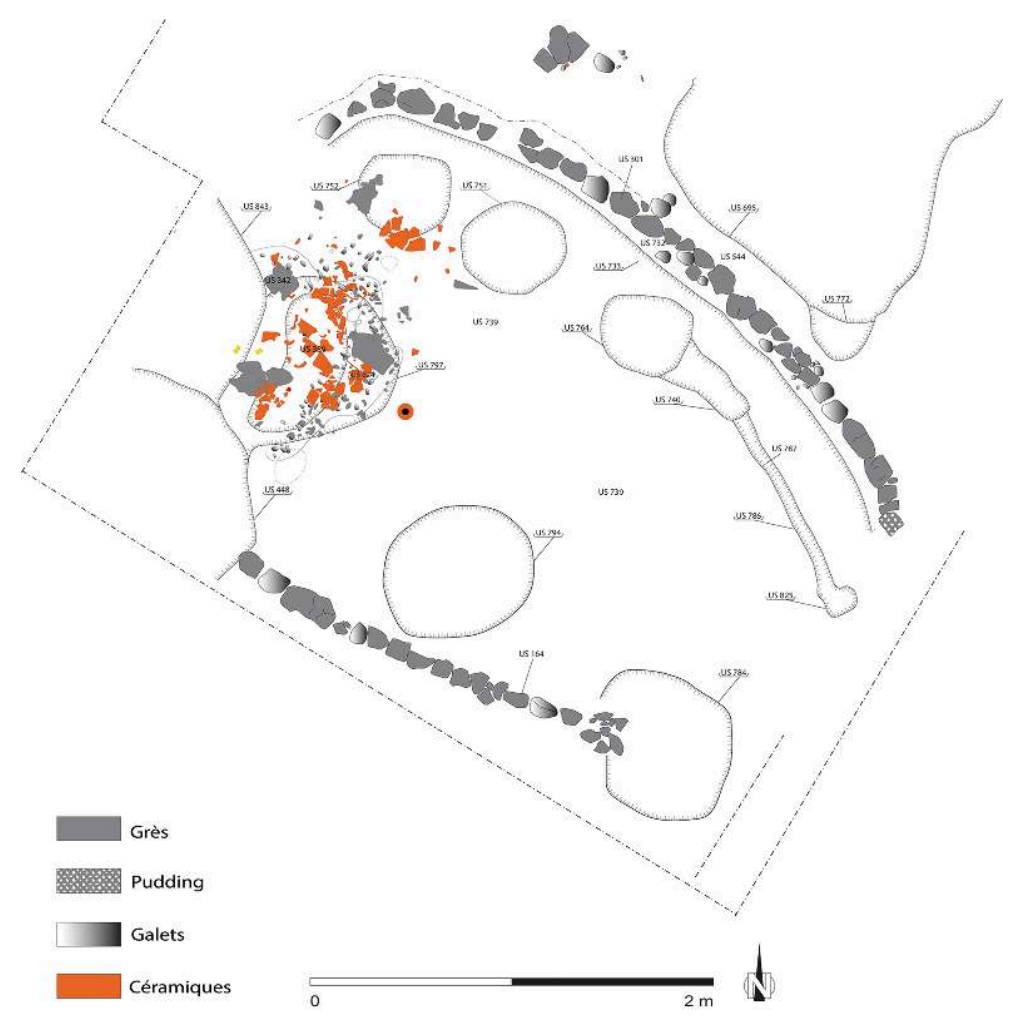

DAO T. Lisa Marchand.

6 Au centre de l'abside - en correspondance significative avec l'espace dépositionnel de la fin du VII siècle - une fosse caractérisée par un profil semicirculaire s'ouvre vers l'ouest (fig. 3, G ; fig. 4, US 797) à la limite du grand creusement qui a démoli la partie postérieure de l'édifice dans la phase d'abandon final du site (fig. 3, H ; fig. 4, US 448, 843). Remplie de terre très foncée, elle a été scellée par un lit de petits galets compactés ; sur le fond, au contact direct avec le terrain vierge, ont été déposés des os et un galet de couleur vert ${ }^{3}$. Comme on peut l'apprécier dans le plan cumulé des fouilles 2013 et 2020 (fig. 4) nous nous trouvons exactement au-dessus de l'espace correspondant à la dernière cérémonie attestée à l'intérieur de l'édifice, selon une pratique qui implique une superposition à la verticale extrêmement précise.

7 L'intérêt considérable de cet aménagement repose sur le fait qu'il nous montre comme dans d'autres contextes mis au jour dans ce secteur de la colline, et comme on le verra également par la suite (fig. 13) - que le volet rituel de l'occupation de cet espace se développe finalement sur plusieurs phases. Plus particulièrement, il atteste une remarquable absence de solution de continuité et une exacte correspondance topographique - matériellement indiquée et visuellement affirmée - entre les pratiques rituelles appartenant à la phase la plus récente et celles réalisées précédemment : ce qui témoigne ultérieurement de la récurrence systématique du phénomène d'une perpétuation intentionnelle d'actions cérémonielles dans un même espace ${ }^{4}$.

L'édifice à abside nous dévoile donc progressivement une histoire bien riche, dont nous avons pu reconnaître - à l'état actuel de nos connaissances - au moins 4 phases de vie, toutes vraisemblablement inscrites à l'intérieur du VII ${ }^{e}$ siècle. Sa vocation rituelle, que la déposition chronologiquement la plus récente nous avait immédiatement dévoilée au moment de sa découverte, a été solidement confirmée par le haut taux de continuité 
des pratiques cérémonielles chtoniennes détectées sur plusieurs niveaux, cumulées l'une sur l'autre à l'intérieur de l'édifice. Ce caractère est d'ailleurs ultérieurement souligné par le fait que le bâtiment a été littéralement encerclé, à l'extérieur, d'un nombre impressionnant de fosses à forte connotation chtonienne (fig. 2).

\section{La grande structure en pierres}

9 En progressant vers l'ouest, nous avons procédé au dégagement de l'espace contournant la grande structure en pierres (fig. 1, B ; fig. 2, B) interceptée lors des campagnes précédentes ${ }^{5}$ et en même temps à la libérer des couches qui la recouvraient partiellement encore, pour essayer d'en déterminer la physionomie et d'en établir une chronologie plus précise.

Nous avons procédé en première instance à la fouille des comblements des fosses qui l'entourent du côté nord-est (la zone actuellement non plus recouverte par les grandes couches d'oblitération finale, en revanche encore présentes sur le côté sud-ouest, fig. 2, D) à la manière d'une véritable "dentelle » se développant autour de l'édifice (fig. 2,5). Ces fosses se distinguent par une physionomie constante: de forme subcirculaire, elles apparaissent couplées deux à deux et strictement adjacentes (fig. 5, $\left.\mathbf{A A}^{1}, \mathbf{B B}^{1}, \mathbf{C C}^{1}\right)$. Les modalités dans lesquelles elles ont été comblées, les procédés de la déposition et les caractéristiques du mobilier présent à l'intérieur (céramique à impasto, indigène, grecque d'importation, fusaïoles), lui confèrent une connotation rituelle prononcée.

Fig. 5. Photogrammétrie de la zone de la grande structure en pierres.

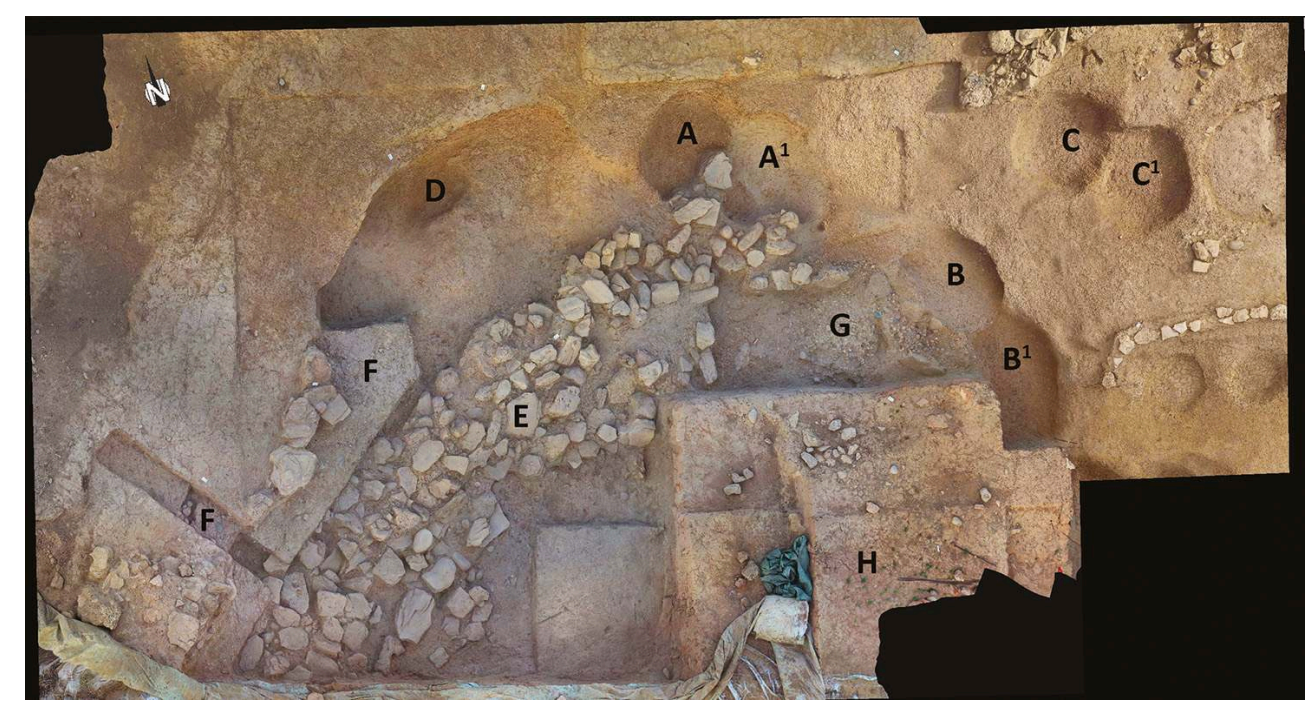

C. Vita.

11 La plus grande de ces fosses, creusée tout au long du côté nord de la grande structure (fig. 1, US 564 ; fig. 5, D), avait été partiellement fouillée en 2018, en révélant dans son comblement un choix sélectif d'objets de prestige, déposés dans un état de conservation exceptionnel: parmi de nombreux os d'animaux, de la céramique matt painted et à impasto, de très nombreux exemplaires de céramique proto-corinthienne d'importation, dont les formes relèvent d'un contexte post-rituel à caractère libatoire 
et sacrificiel (des amphores - dont une, corinthienne, déposée entière, des skyphoi et des kotylai, un krateriskos, une œnochoé, une chytra, un louterion) ${ }^{6}$.

La progression de la fouille a permis de saisir presque définitivement les limites de cette importante déposition, caractérisée par la présence, grosso modo à la moitié de sa réalisation, d'une remarquable concentration de gros galets (fig. 6, B), aussi bien que d'une énorme pierre (fig. 6, C). Elle nous a conduit également à récupérer le reste du mobilier qui y avait été déposé, afin de compléter ultérieurement nos connaissances sur cet extraordinaire ensemble de céramique - notamment d'importation corinthienne (fig. 7) - partiellement mise au jour en 2018. Au sein de ce lot sont à remarquer les fragments d'un stamnos peint de production locale (fig. 8), appartenant à une classe de céramique normalement attestée à Incoronata dans les grands dépôts fouillés par l'Université de Milan et de Rennes, dont la chronologie peut être établie génériquement à la fin du $\mathrm{VII}^{\mathrm{e}}$ siècle, mais dont nous connaissons encore mal les contextes stratigraphiques. Dans ce cas, en revanche, ce document pourra être inscrit de manière bien plus précise à l'intérieur d'une grille chrono-stratigraphique certaine, nous fournissant des informations primordiales pour la connaissance de la dernière phase d'utilisation de cette poterie à destination cérémonielle, réalisée sur place par des céramistes grecs immigrés?

Fig. 6. La fosse au nord de la structure en pierres, en cours de fouille.

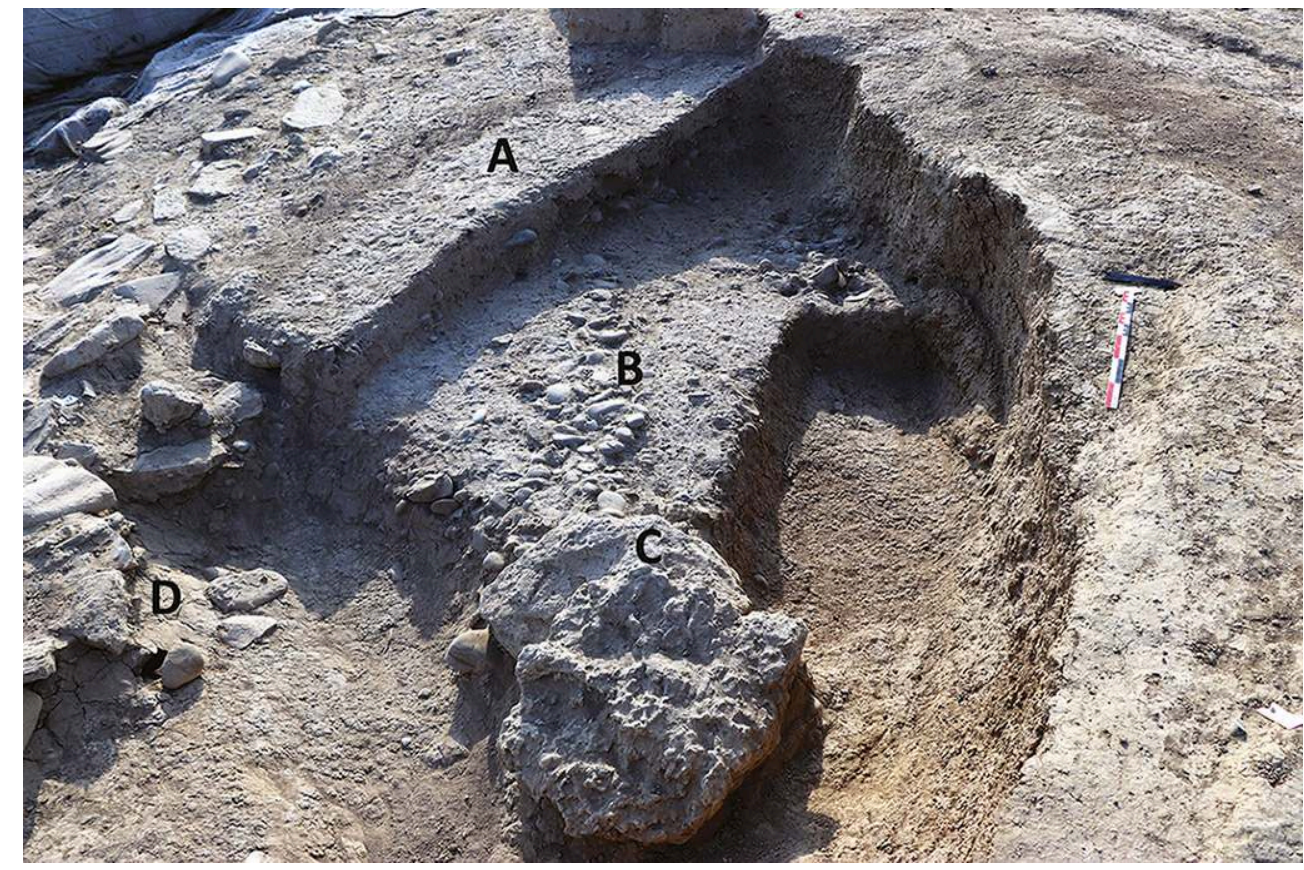

M. Denti. 
Fig. 7. Céramique grecque d'importation, du remplissage de la fosse à la fig. précédant.

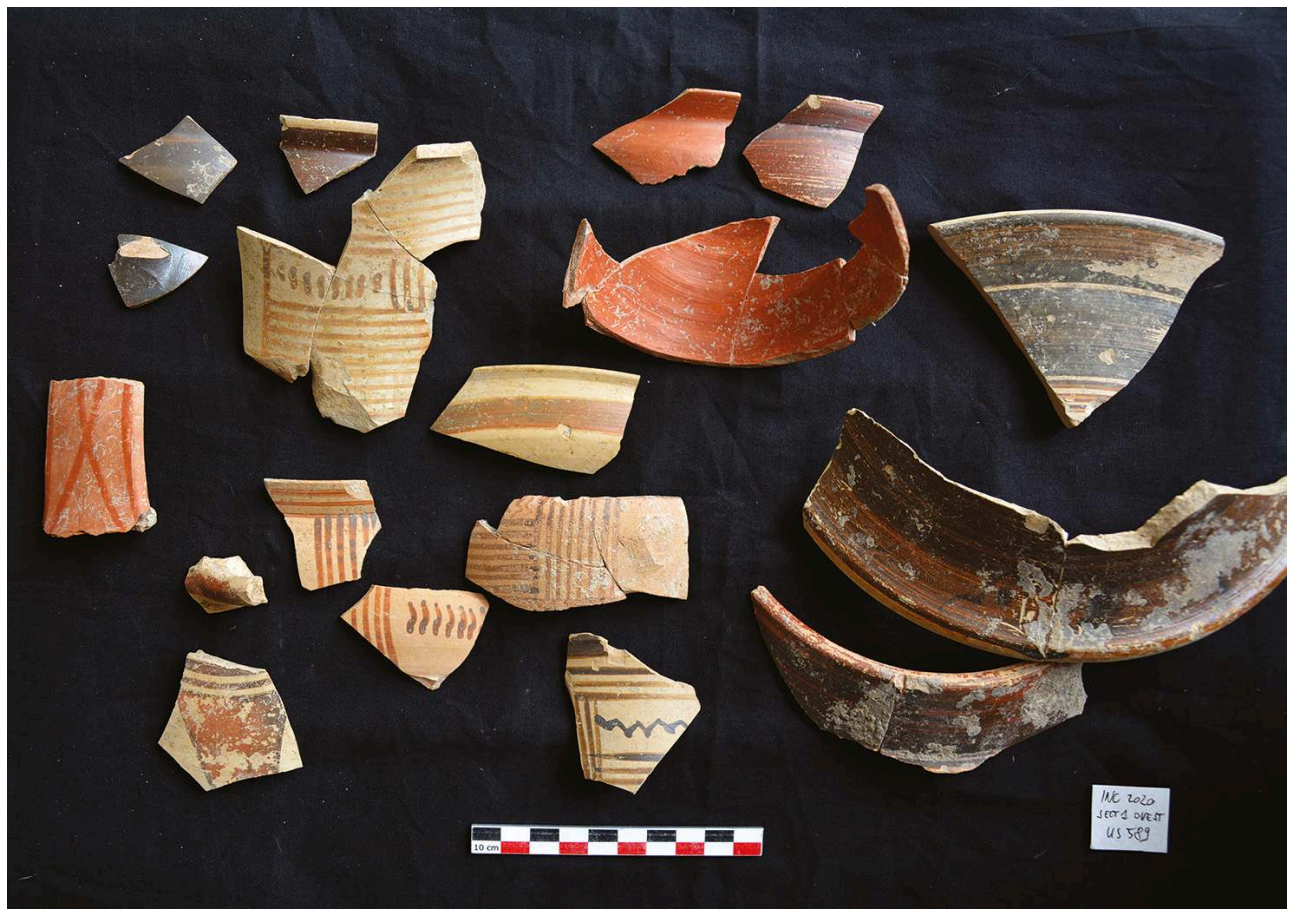

M. Denti.

Fig. 8. Fragments d'un stamnos de production locale, du remplissage de la fosse à la fig. 6 .

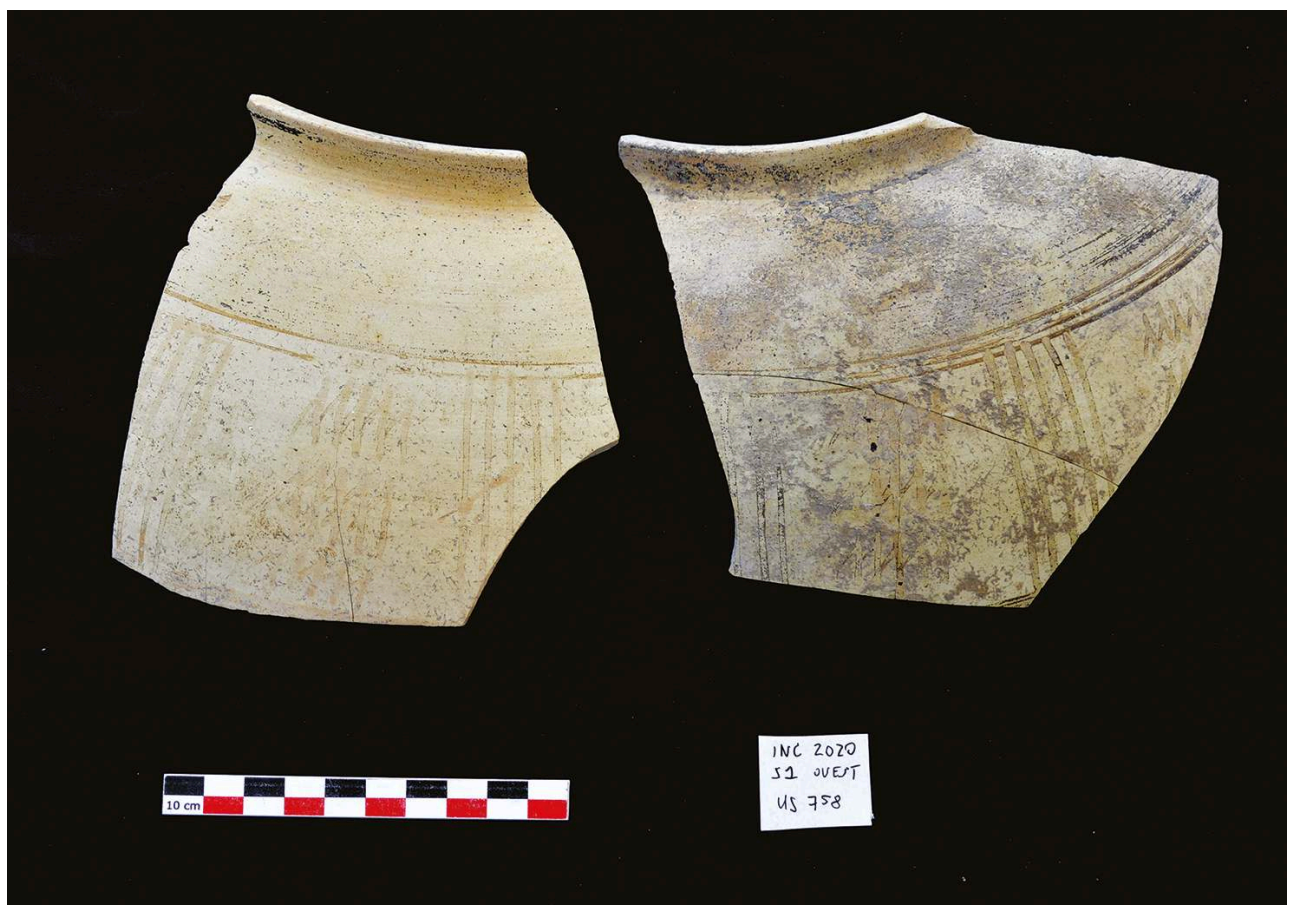

M. Denti.

13 La donnée la plus importante concernant ce contexte réside en effet dans l'acquisition d'une séquence chronologique fiable et ancrée avec précision au double volet de la stratigraphie et de la datation de la céramique grecque d'importation associée à ces 
couches, susceptible de pouvoir commencer à dater la grande structure en pierres. De haut en bas, nous avons donc la séquence suivante :

14 1) Le pavement en cailloutis très fins, qui se situe tout au long de la limite nord de la structure en pierre, et qui en couvre partiellement le bord (fig. 1, US 640 ; fig. 5, F ; fig. 6, A ; fig. 10) peut être daté à l'intérieur de la deuxième moitié du VII ${ }^{e}$ siècle. Il s'agit d'une chronologie d'ailleurs bien correspondante à celle de la petite tête dédalique en bronze qui gisait sur celui-ci, découverte en $2018^{8}$.

2) Le comblement de la fosse (on en voit encore une partie, en cours de fouille, dans la fig. 6, B) a été réalisé au-dessous de ce sol : il en est donc plus ancien. Cette relation stratigraphique se voit confirmée par la chronologie de la céramique grecque importée contenue dans le comblement (fig. 7) : nous sommes autour du milieu du VII ${ }^{\mathrm{e}}$ siècle?

16 3) Ce comblement s'appuie à la paroi septentrionale (fig. 6, D) de la grande structure réalisée en terre et pierres. Celle-ci, par conséquence, est très vraisemblablement préexistante au comblement. Cette relation chrono-stratigraphique nous a été d'ailleurs confirmée, de manière significative et cohérente, par l'attestation exclusive de céramique figurée matt painted, de très haute qualité formelle et de datation haute (fig. 9), retrouvée parmi les blocs de pierre qui constituent le parement supérieur de la structure : elle se révèle donc assez ancienne, pouvant s'accrocher à la fin du VIII ${ }^{\mathrm{e}}$ début du VII siècle.

17 La conclusion que nous pouvons tirer est simple et à la fois très importante. Cette impressionnante structure n'est pas récente, elle est plutôt ancienne. Elle ne semble pas appartenir au plein VII ${ }^{e}$ siècle, mais remonter vers le début de celui-ci : elle pourrait correspondre, en effet, aux grands travaux édilitaires qui ont caractérisé l'occupation œnôtre du site pendant le VIII siècle - quand les Grecs n'étaient pas encore présents - et sont attestés par les structures déjà mises au jour dans la partie méridionale de ce secteur de la colline. Il s'agit de pavements, de structures bâties en pierres et terre, significativement caractérisés par des techniques architecturales tout à fait analogues (si non identiques: comparons ce sol US 640 au sol US 38 - «PV 1 », au sud) ${ }^{10}$ à celles maintenant découvertes plus au nord. 
Fig. 9. Céramique matt painted monochrome figurée, retrouvée à l'intérieur de la structure en pierres.

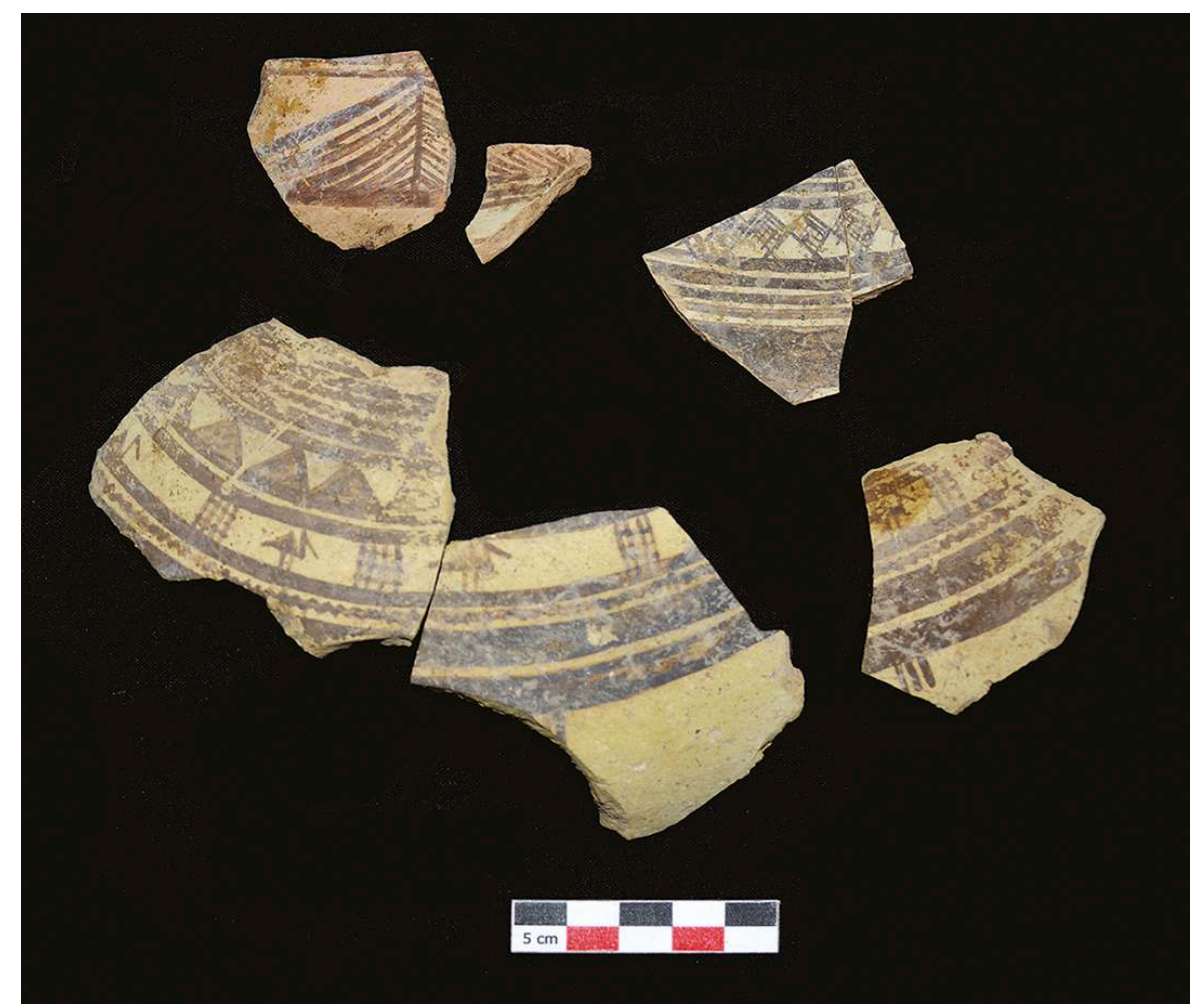

M. Denti.

Sur le côté occidental de la structure nous avons ouvert un petit sondage qui a coupé, en sens nord/ouest - sud/est, les couches de recouvrement de la structure. Ici, il a été possible d'intercepter le prolongement du sol en question, aussi bien que sa limite, vers le nord (fig. 1-2 ; fig. 5, F); il a été construit sur une strate sableuse de préparation. La prochaine campagne aura pour objectif de cerner la limite de son profil septentrional.

Sur le côté opposé de la structure, à l'est, nous devons signaler l'existence d'une intéressante, mais encore énigmatique, concentration de micro galets, auxquels sont associées des pierres formant un angle (fig. 1, US 817 ; fig. 5, G; fig. 11). Cette concentration, extrêmement dure et compacte, était recouverte d'une strate d'argile (US 834) contenant des petits charbons de bois et une importante concentration de micro tessons appartenant à de la céramique achrome, de grands conteneurs, de l'impasto et, au moins, à 3 skyphoi d'importation PC.

La grande structure monumentale atteint actuellement une dizaine de mètres de long et jusqu'à $2,80 \mathrm{~m}$ de large. Elle est réalisée en pierres de forme irrégulière, aménagées à l'intérieur d'un lit de terre ; une bonne partie de ces pierres est posée à plat (fig. 5, 10). L'état de l'avancement de la fouille ne nous autorise pas encore à en décrire la fonction. Prenons toutefois en considération un nombre non négligeable d'éléments dont nous disposons : la technique architecturale; la très probable association avec les briques réemployées in situ dans les couches de l'oblitération définitive de l'édifice, qui auraient bien pu servir comme éléments de son élévation; les dimensions imposantes de l'artefact; l'association à des dépositions rituelles de très grand prestige (céramiques grecque d'importation, sculpture en bronze); le fait que la structure apparaît systématiquement encerclée par une série de fosses rituelles; enfin, l'impressionnant 
engagement développé (en termes de main d'œuvre, de moyens, de technique, de matériel) pour mettre en œuvre sa démolition - et sa conservation dans la mémoire grâce à l'une des plus gigantesques oblitérations recensées par l'archéologie méditerranéenne pour l'âge du Fer (fig. 2, D ; fig. 5, H ; fig. 10-11) ${ }^{11}$. Ces arguments suggèrent, avec beaucoup de vraisemblance, l'attribution de la structure à la fondation d'un édifice doté de dimensions considérables et d'une signification particulièrement remarquable.

Fig. 10. La grande structure en pierres, vue de l'ouest.

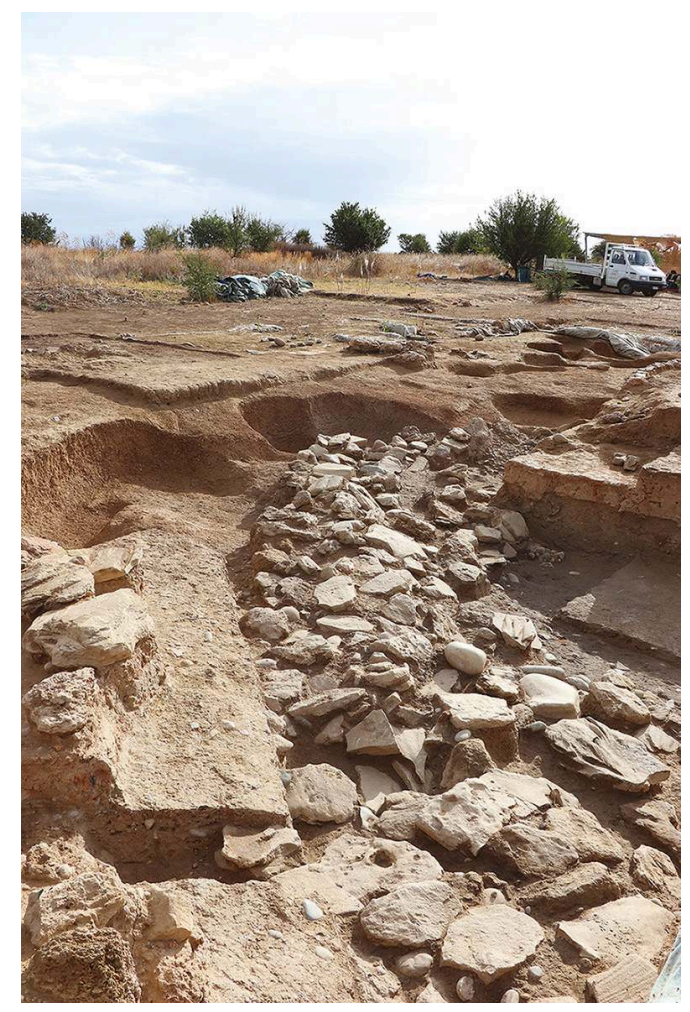

Sur la gauche de l'image (au nord), la partie restante du sol US 640, qui recouvrait la structure en pierres tout au long de son côté septentrional ; sur la droite (au sud), les grandes couches de l'oblitération du monument, non encore enlevées.

M. Denti. 
Fig. 11. La structure en pierres vue du nord-est, avec les fosses qui l'entourent au nord, les strates du gigantesque recouvrement oblitérant au sud et, à l'extrême gauche de l'image, la concentration de galets US 817.

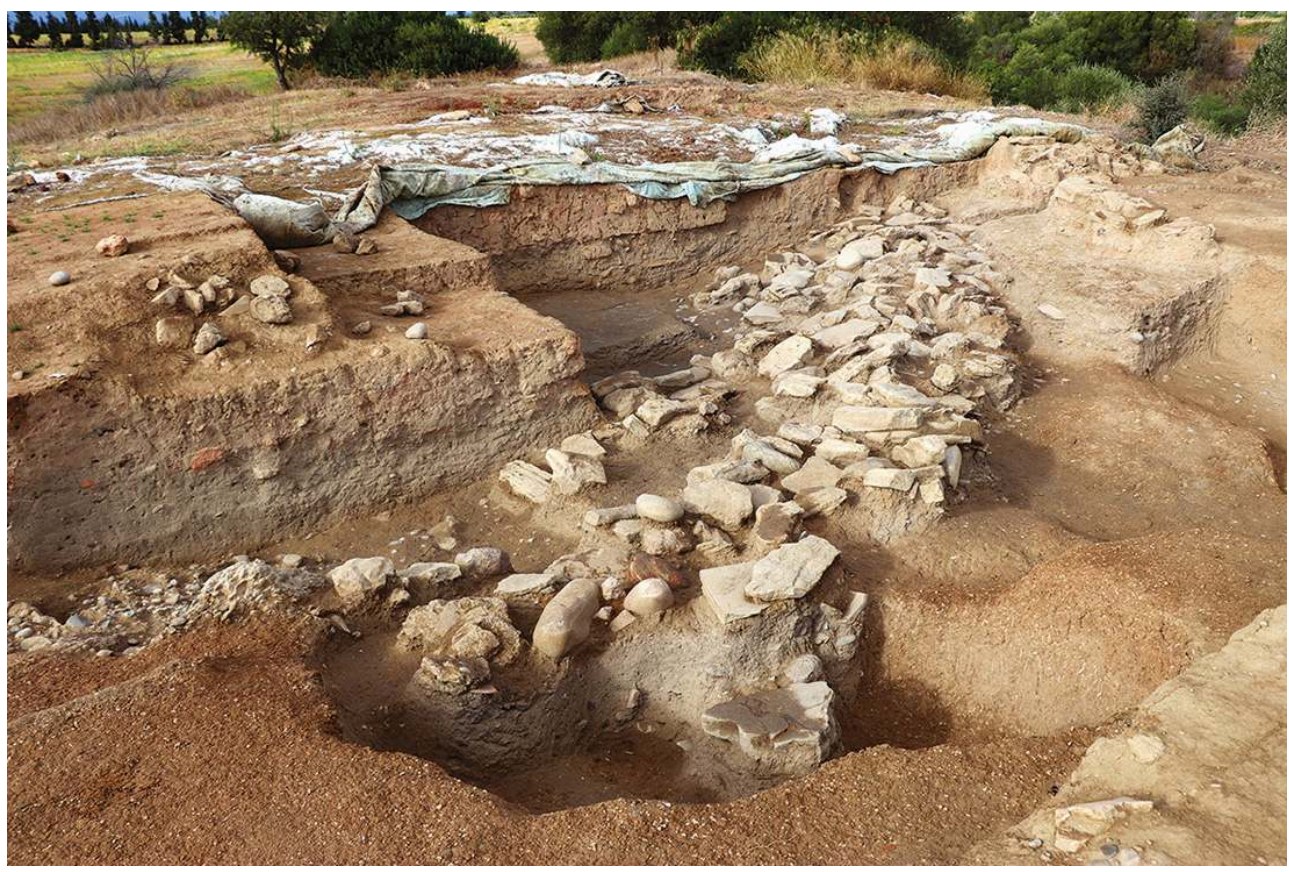

M. Denti.

21 Seule l'ablation de l'énorme strate d'oblitération en terre et briques qui encore protège cette structure vers le sud (fig. 2, D ; fig. 5, H ; fig. 10-11) - opération prévue pour la campagne 2021 - permettra probablement d'en saisir la réelle nature, ou au moins de nous approcher ultérieurement de cet objectif. À l'heure actuelle, cette spectaculaire construction représente un nouveau témoignage extraordinaire du niveau de l'activité à caractère monumental réalisée sur ce site par les élites indigènes de la Basilicate à l'âge du Fer, en confirmant la centralité de la fonction hégémonique du site d'Incoronata dans l'horizon historique de l'occupation de l'€nôtrie côtière.

\section{La fosse au nord}

22 À la limite septentrionale de la zone de fouille (fig. 1, C ; fig. 2, C) nous avons procédé à l'exploration des niveaux situés au-dessous du contexte rituel mis au jour en 2019, apparu à l'origine complètement recouvert - c'est-à-dire protégé- par une remarquable accumulation de gros galets ${ }^{12}$. Cet aménagement rituel était constitué par un lit de galets sur lequel des restes de repas et de libations gisaient (des ossements, le crâne d'un veau de quelques mois d'âge, des tessons appartenant à des coupes grecques datables du VII siècle), encadrant du côté nord une structure concave, avec un profil ovale, scellée par des galets posés sur une couche d'écailles de pierres et de briques (possible « bouchon » d'un bothros) ${ }^{13}$ (fig. 12, A ; fig. 1 et 13, US 735, 763 ; fig. 14). 
Fig. 12. Photogrammétrie de la zone nord de la fouille.

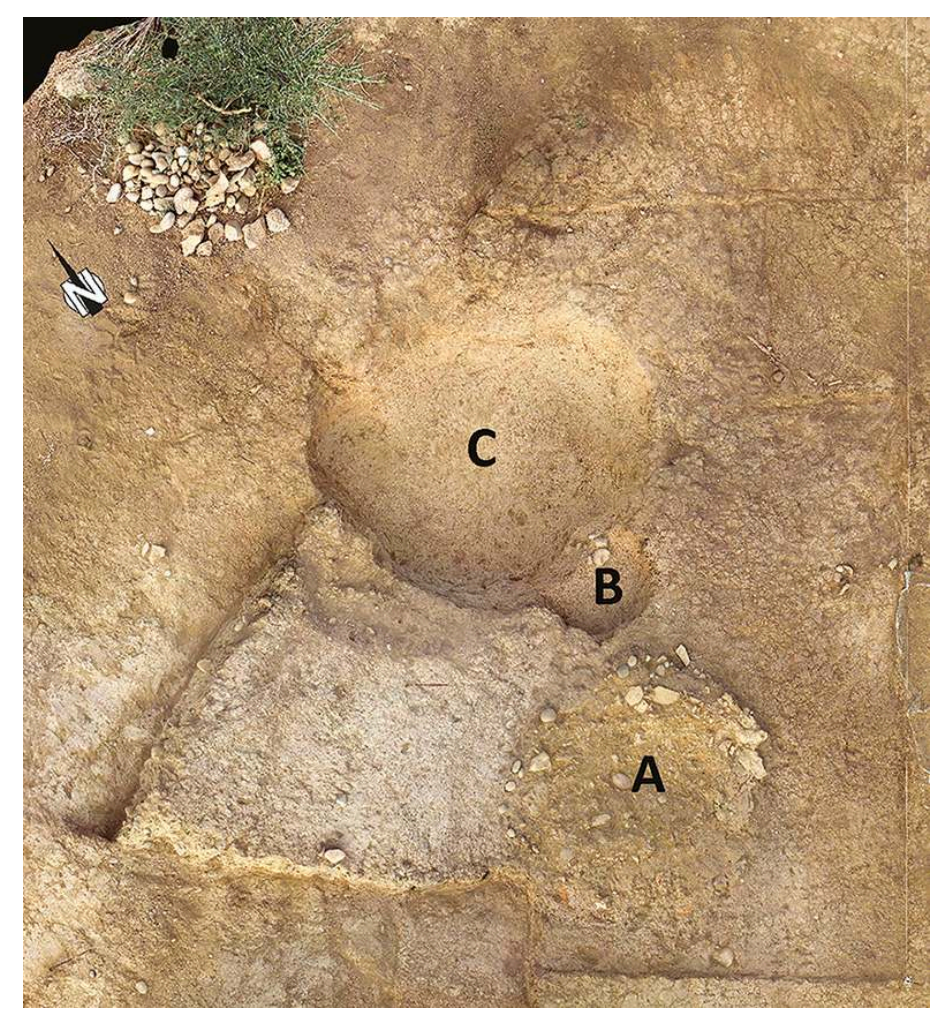

C. Vita.

23 Exactement sous cet espace dépositionnel, juste à côté du bothros supposé, une petite fosse circulaire est apparue (fig. 1 et 13, US 799 ; fig. 12, B ; fig. 14). Le plan cumulé des fouilles 2019 et 2020 dans ce secteur (fig. 13, US 748 sur US 799) nous montre de manière extrêmement efficace cette superposition. La petite fosse présentait à nouveau, dans la partie haute, un scellage constitué par un lit de petits galets (fig. 14) ${ }^{14}$ et contenait, entre autres, une coupe cantharoide d'importation PC datable de la première moitié du VII ${ }^{e}$ siècle. Voici un nouveau cas (après celui que nous venons d'observer à l'intérieur de l'édifice à abside, fig. 4) témoignant d'une précise - et explicite - continuité rituelle, réalisée dans un même espace sur plusieurs moments de la fréquentation du site. 
Fig. 13. Planimétrie cumulée 2019 e 2020 de la zone nord de la fouille 2020.

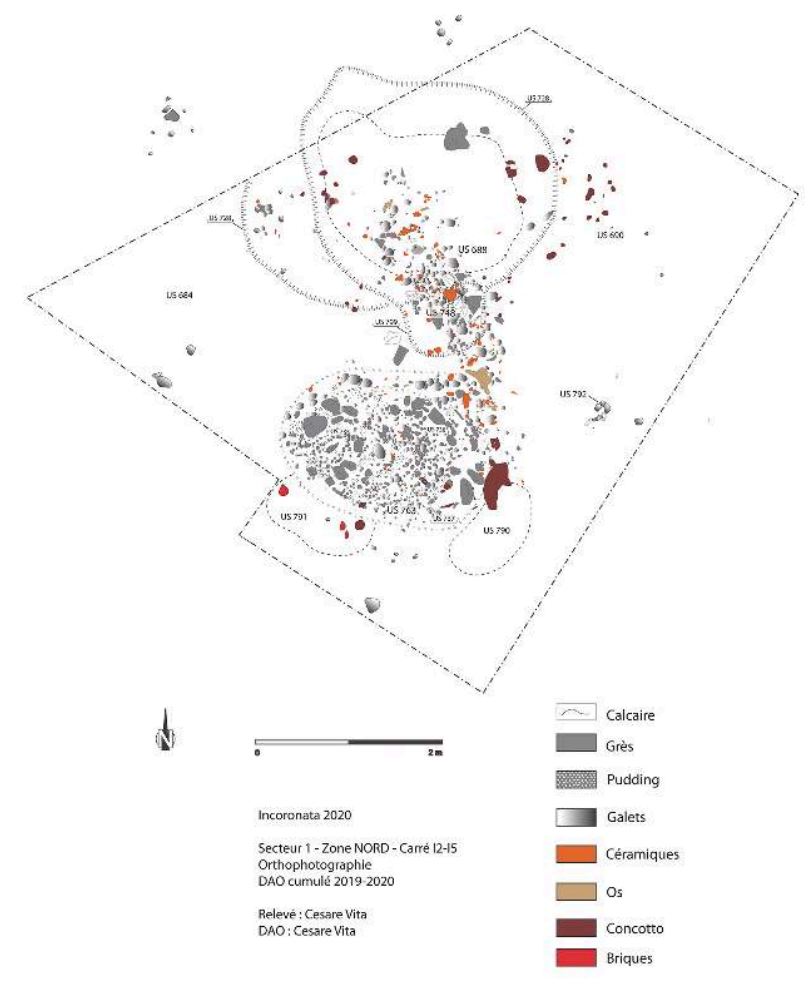

DAO T. Lisa Marchand

Fig. 14. Détail de la zone nord, vue de l'est, au début de la fouille.

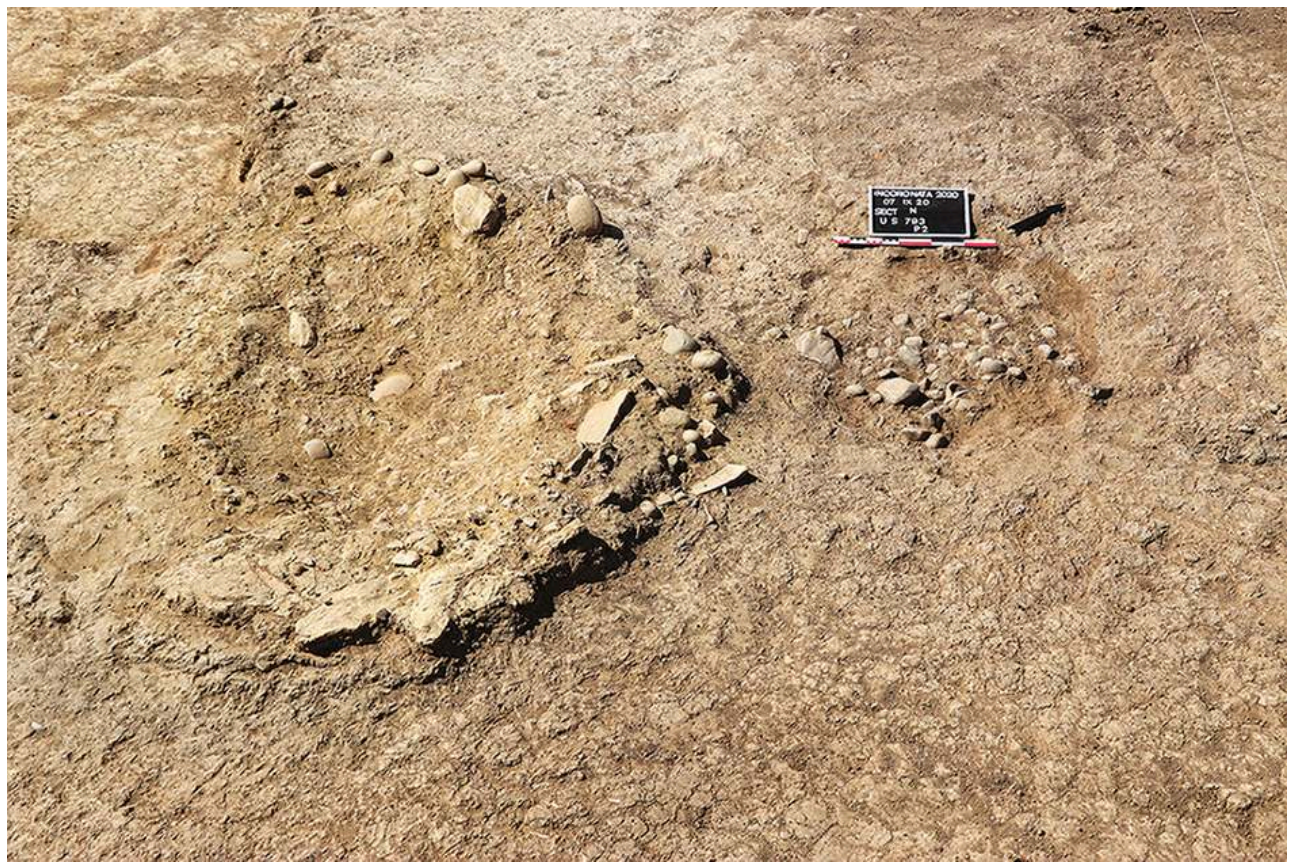

Sur la gauche de l'image, la structure concave ovale ; sur la droite, la petite fosse US 799 scellée par un lit de petits galets.

Cette petite fosse avait été creusée à l'intérieur de la limite méridionale d'une bien plus grande fosse de forme ovale, mesurant $\mathrm{m} 2,50 \times 2,90$ (fig. 1 et 13, US 728 ; fig. 12, C), 
dont le comblement comprenait du matériel particulièrement remarquable par quantité, qualité et intérêt scientifique : parmi d'importantes quantités d'impasto, de la céramique indigène (monochrome et bichrome) et grecque (locale et d'importation), un grand conteneur intentionnellement déposé presque entier, des pesons (dont un décoré), une lame en fer, des fragments appartenant aux parois et à la sole d'un four. De nombreuses portions d'adobe avaient été rejetées à l'intérieur d'une couche de 13-15 cm, se trouvant au centre du remplissage de la fosse (US 826), pleine de cendre, avec des charbons de bois et de morceaux de la carapace d'une tortue. Sous cette couche gisaient, enfin, 3 pierres associées à la déposition de nombreux tessons de céramique matt painted, achrome, d'impasto, et d'un autre peson (US 829, fig. 15): les pierres avaient été posées directement sur le fond du creusement, constituant le point initial du comblement.

Fig. 15. Céramique matt painted et pesons, des couches du comblement de la grande fosse dans le secteur nord de la fouille.

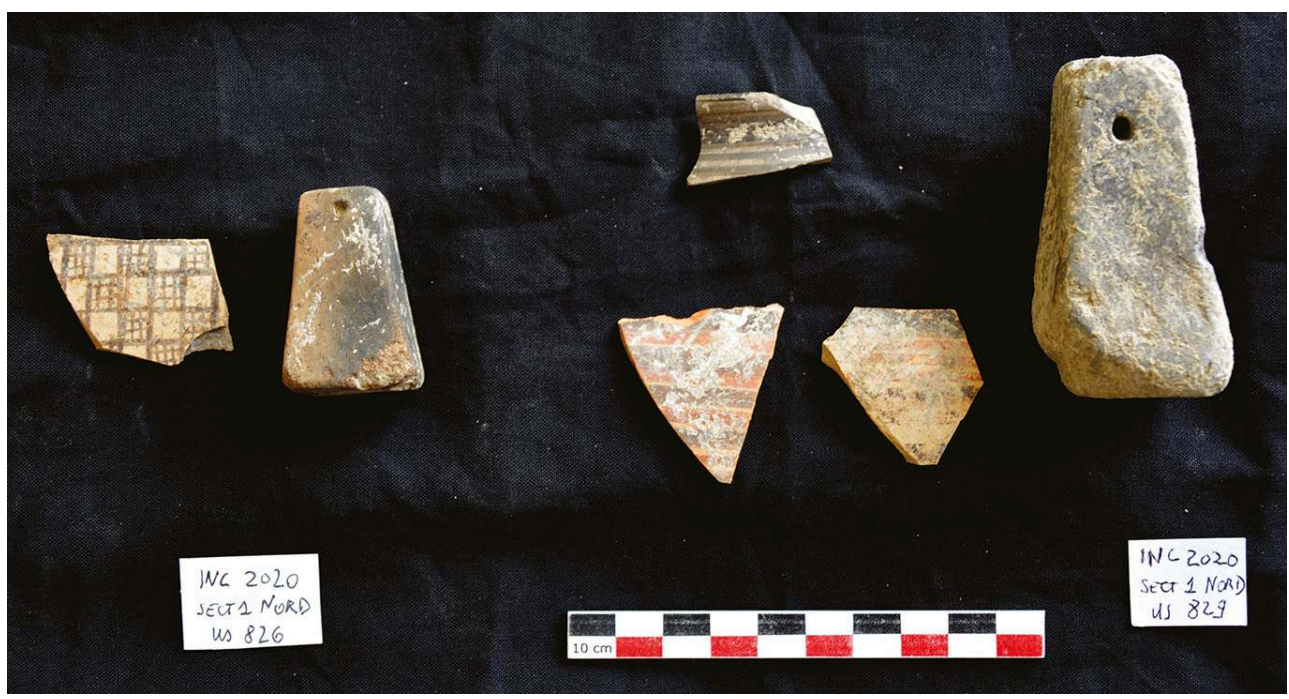

M. Denti.

La nouvelle fosse interceptée et fouillée cette année se trouve un peu plus au nord par rapport aux autres nombreuses fosses qui contournent l'édifice à abside et la grande structure en pierres (fig. 2) et se signale par ses dimensions, bien plus grandes que la moyenne des autres. Des éclaircissements ultérieurs concernant sa nature et sa fonction nous seront certainement fournis par la fouille - prévue pour la campagne 2021 - de l'autre (possible) bothros adjacent au sud, mais le caractère décidément ritualisé des actions réalisées tout au long de son remplissage ne laisse pas de doutes sur la connotation cérémonielle de cette opération.

\section{Conclusions}

À l'issue de la campagne de fouille 2020, nous pouvons considérer que nous sommes proches de la compréhension définitive de l'un des espaces-clé de l'occupation de la colline d'Incoronata. Dans l'état actuel de l'avancement de la recherche, n'est en effet plus mise en question la nature de cette occupation - désormais suffisamment claire, et sur laquelle nous avons abondamment écrit - mais de reconnaître de manière plus 
approfondie le fonctionnement réciproque des différents éléments mis au jour, dans l'espace comme dans le temps. De compléter, en un mot, un puzzle de longue date, que seule une fouille par nécessité particulièrement fine et lente, comme celle-ci, permet d'appréhender dans son intégrité.

Une clé incontournable pour ouvrir cette perspective sera l'enlèvement intégral de la partie résiduelle de la grande couche d'oblitération, encore in situ au centre de ce secteur (fig. 11), comme on peut clairement l'observer sur le plan actuel de la fouille (fig. 1). S'il ne faut pas oublier que c'est grâce à cette spectaculaire protection intentionnelle - réalisée au moment de l'abandon définitif du site (fin VII ${ }^{e}$-début $\mathrm{VI}^{\mathrm{e}}$ siècle) - que nous avons pu mettre au jour un faciès archéologique caractérisé par un état de conservation vraiment exceptionnel, elle empêche actuellement de saisir le cœur de la question, car elle recouvre le reste du grand bâtiment que nous venons de dévoiler. Son ablation nous permettra, en reliant les structures du nord et du sud de cet espace, d'en libérer le centre : un centre où l'épaisseur particulièrement imposant de cette oblitération nous laisse entendre l'envergure des données sous-jacentes.

Si nous observons d'en haut la topographie de la zone qui a fait l'objet de l'exploration de cette année (fig. 2), il semble désormais évident que tous ces contextes rituels - des dizaines de fosses, avec leurs plans de dépositions post-cérémonielles - sont positionnés de manière extrêmement cohérente tout autour de l'édifice à abside, aussi bien que de la grande structure en pierres qui est en train de revoir le jour: certainement l'un des monuments majeurs, peut-être le réel centre névralgique de l'occupation du site.

\section{BIBLIOGRAPHIE}

DENTI 2013

Mario Denti, « Incoronata. Les résultats de la dixième campagne de fouille (2012). Ruptures et continuités dans l'occupation du site entre VIII ${ }^{\mathrm{e}}$ et VII ${ }^{\mathrm{e}}$ siècle avant J.-C. », Chronique des activités archéologiques de l'École française de Rome, Italie du Sud, en ligne, http://journals.openedition.org/ cefr/878, consulté le 29 janvier 2021.

DENTI 2014

Mario Denti, « Incoronata, la onzième campagne de fouille (2013). Les structures de l'âge du Fer, des composants de l'espace artisanal, un édifice absidé à vocation rituelle », Chronique des activités archéologiques de l'École française de Rome, Italie du Sud, en ligne, http://journals.openedition.org/ cefr/1096, consulté le 29 janvier 2021.

DENTI 2018

Mario Denti, « Des pratiques rituelles perpétuées sur deux siècles d'occupation à Incoronata. La campagne de 2017 », Chronique des activités archéologiques de l'École française de Rome, Italie du Sud, en ligne, http://journals.openedition.org/cefr/1928, consulté le 29 janvier 2021.

DENTI 2018a

Mario Denti, « Archilochos did not Sail Alone to the Bountiful Shores of Siris: Parian and Naxian 
Potters in Southern Italy in the 7th Century BC. ", in Dora Katsonopoulou (éd.), Paros IV. Paros and its Colonies, Fourth International Conference on the Archaeology of Paros and the Cyclades (Paros

11-14 June 2015), Athènes, The Institute for Archaeology of Paros \& the Cyclades, 2018, p. 39-63.

DENTI 2019

Mario Denti, «Cultes et pratiques rituelles chtoniens à Incoronata. Campagne 2018 ", Chronique des activités archéologiques de l'École française de Rome, Italie du Sud, en ligne, http:// journals.openedition.org/cefr/3274, consulté le 29 janvier 2021.

DENTI 2019a

Mario Denti, « A bocca aperta. Una piccola scultura dedalica in bronzo in un contesto cultuale indigeno dell'Italia meridionale », in F. Vistoli (éd.) Miscellanea di studi in memoria di Enzo Lippolis I, AMSMG Va serie, IV, 2019, p. 65-83.

DENTI 2020

Mario Denti, « Des serpents à Incoronata. La campagne de 2019 », Chronique des activités archéologiques de l'École française de Rome, Italie du Sud, en ligne, http://journals.openedition.org/ cefr/4562, consulté le 29 janvier 2021.

DENTI sous presse

Mario Denti, «Ritual pebbles. Pebbles between the living and the dead in the Mediterranean Iron Age », in John Boardman, James Hargrave, Alexandru Avram, Alexander Podossinov (éd.) Connecting Ancient West and East. Studies presented to Prof. Gocha Tsetskhladze, Louvain 2021, p. 963-1011, sous presse.

\section{NOTES}

1. DENTI 2014, p. 12, fig. 15-19. NB : toutes les dates sont avant J.-C.

2. DENTI 2020, p. 4, fig. 4-5.

3. Sur les procédures de la déposition des galets et ses significations, DENTI 2020, sous presse.

4. DENTI 2018.

5. DENTI 2019, p. 13, fig. 15-23 ; DENTI 2020, p. 16, fig. 19-23.

6. DENTI 2019, p. 16, fig. 15-21.

7. DENTI 2018a. Sur cet argument l'auteur de ces lignes est en train de terminer la rédaction d'une monographie consacrée à l'étude de la céramique grecque figurée de production locale d'Incoronata et de la côte ionienne de l'Italie méridionale du VII ${ }^{\mathrm{e}}$ siècle.

8. DENTI 2019, p. 19, fig. 22-23 ; DENTI 2019a.

9. Une précision ultérieure sera obtenue suite à l'étude de la céramique grecque d'importation et de production locale de ce secteur, actuellement en cours par Josipa Mandić.

10. DENTI 2013, p. 5, fig. 5-19 ; DENTI 2014, p. 6, fig. 7-11.

11. DENTI 2020, p. 18, fig. 19-21.

12. DENTI 2020, p. 11, fig. 13.

13. DENTI 2020, p. 12, fig. 14-16. 
14. DENTI 2020, fig. 14.

\section{INDEX}

chronologie https://ark.frantiq.fr/ark:/26678/pcrtW9SpIgIk7Q

Année de l'opération : 2020

Thèmes : $\mathrm{CJB}$

sujets https://ark.frantiq.fr/ark:/26678/pcrt4StkSBEvk6, https://ark.frantiq.fr/ark:/26678/

pcrtPEkp3Yydb2, https://ark.frantiq.fr/ark:/26678/pcrthBOo082xvY, https://ark.frantiq.fr/ark:/ 26678/pcrtX04WWmXAHL, https://ark.frantiq.fr/ark:/26678/pcrts62Z2hl4ux

lieux https://ark.frantiq.fr/ark:/26678/pcrt071cMeVHkd, https://ark.frantiq.fr/ark:/26678/ pcrtrU1sOVZ1Wo

\section{AUTEURS}

\section{MARIO DENTI}

Laboratoire LAHM, UMR 6566 CReAAH, Université Rennes 2, IUF 